University of Reading: "German Life and Letters", Bd.50, April 1997, S. 133-154

\title{
Die Wende in Deutschland im Spiegel der zeitgenössischen deutschen Literatur
}

\section{Von Hans-Georg Soldat}

r

ielleicht sollte es einem Literaturkritiker, einem in gewisser Weise Fachfremden also, gestattet sein, eingangs festzustellen, daß er in seiner Arbeit nicht primär darauf achten darf, ob in Büchern bestimmte Themen aufgegriffen und in welcher Hinsicht behandelt werden. Vielmehr muß er an der Frage interessiert sein, ob Sujet und literarische Form jene Einheit bilden, die gute Literatur erst konstituiert. Es dürfte daher kaum verwundern, daß es ihm in der Vorbereitung dieses Beitrags schien, als formuliere die Tagung ein Thema, das der verhängnisvollen >Soziologisierung` kultureller Phänomene über Gebühr Vorschub leistet. Natürlich darf man bei solcher Skepsis nicht stehen bleiben. Die Einbeziehung aktueller politischer und gesellschaftlicher Entwicklungen in den Korpus der Literatur ist nicht nur eine Forderung doktrinärer Ideologien, die damit die >Erziehung des Menschen $<\mathrm{zu}$ befördern trachten, sondern ebenso Kennzeichen der freien Literatur.

Gleichsam zum Ausgleich solch anfänglicher Skepsis habe ich mich bemüht, ein paar kaum bekannte Veröffentlichungen in diese unvollständige Übersicht einzubeziehen - Kritiker sollten schließlich auch ein bißchen Entdecker sein. Oft werden ja manche Autoren nur deshalb so wenig gewürdigt, weil sie keine berühmten Namen tragen oder weil ihre Bücher an eher entlegener Stelle erschienen, in kleinen Verlagen oder jenen mittleren der neuen Bundesländer, denen selbst sieben Jahre nach dem faktischen Ende der DDR eine vernünftige >Public Relation< unbekannt ist. ${ }^{1}$ 


\section{Allgemeines zum Thema 'Wender in der Literatur}

Schon eine allererste grobe Sichtung ergibt, daß das Thema $>$ Wende (der Begriff sei in Zukunft ohne die Anführung geschrieben) in ganz überwiegendem Maße von ehemaligen DDR-Autoren aufgegriffen wird. Das hat vielfältige Ursachen, doch der Hauptgrund ist zweifellos, daß sie von dieser Wende existentiell betroffen wurden - sie verloren zumeist ihre garantierte Lebensgrundlage und zudem ihre gesellschaftliche Reputation, ja Macht. Ein genauerer Blick zeigt weiter, daß die Wende meist nicht als Ereignis wahrgenommen oder zumindest dargestellt wird, das genau lokalisiert oder präzise zeitlich definiert werden kann. Was Wende ist, hat in der Literatur eine enorme Spanne; mir scheint die Schlußfolgerung erlaubt, daß Wende eher als Proze $\beta$ denn als etwas Punktuelles empfunden wird. Entsprechend überwiegen in der Literatur indirekte Beschreibungen: Wende als Aufhebung geographischer Trennungen (bei Christa Schmidt in Rauhnächte ${ }^{2}$ ) und noch mehr örtlicher Einschränkungen (Irina Liebmann in ihrem Roman In Berlin $^{3}$ ). Wende weiter als gewissermaßen $>$ Vorher-Nachher $<-B e t r a c h-$ tung, als Räsonnement, als verbale Auseinandersetzung mit der eigenen Rolle im gesellschaftlichen Geflecht der früheren DDR. Volker Braun hat diese Form in der >Unterhaltung « (wie er es selbst nennt) Der Wendehals $^{4}$ vielleicht am reinsten dargestellt. Daniela Dahn wählt in ihren Essays eine Mischform aus beiden. Auch Marion Titze wäre zu nennen, die mit Unbekannter Verlust $t^{5}$ ebenfalls in diese Kategorie gehört.

Besonders diffizil wird das Thema, sobald es um die >Stasi< geht, das Ministerium für Staatssicherheit der DDR, genauer den berühmtberüchtigten $>\mathrm{IM}<$, den `Inoffiziellen Mitarbeiter८, ein Trauma unterdessen auch für viele, die sich des Themas Wende in der Manier des literarischen >Vorher-Nachher anzunehmen versuchen. Hier verschränken sich handelnde Personen (Fritz Rudolf Fries, selbst als IM >Pedro Hagen wohl Spitzel der Stasi - um nur ein Beispiel zu nennen), Roman-Sujet (Wolfgang Hilbigs Figur des >Cambert in $\gg I c h \aleph^{\circ}$ ) und gelungene oder mißlungene Untersuchungen in geradezu exemplarischer Weise. Das reicht von einer falschen Dämonisierung der Stasi über Versuche der belletristischen Objektivierung (bei Uwe Kolbe, geb. 
1957 in Berlin), der dies etwa in seinem Essay $>$ Die Situation ${ }^{7}$ in einzelnen Artikeln, die in verschiedenen Zeitungen und Zeitschriften ${ }^{8}$ erschienen und - schon etwas verschlüsselter - in einem neueren Text, >Drei Episoden aus dem kalten Frieden,${ }^{9}$ versuchte, und es endet bei gefährlichen Geschichtsklitterungen (〉Geheimdienste sind überall schlimm ...८).

In den meisten Büchern ist >die Wende< freilich einfach geschehen, sie wird vorausgesetzt, ohne ihr mehr als die bloße Existenz zuzubilligen (Jens Sparschuh, Der Zimmerspringbrunnen, ${ }^{10}$ aber auch teilweise Angela Krauß in Die Überfliegerin ${ }^{11}$ ). Mir scheint darin, gerade angesichts der deutschen Erfahrungen nach 1945, wo man ähnliche Tendenzen in der Literatur beobachten konnte, purer Eskapismus zu stecken. So, wie damals der Krieg zzu Ende war`, und man simplifizierend von $>$ der Niederlage Deutschlands` sprach, ist nun >die Wende` über OstDeutschland gekommen, und wieder in ähnlichen Konnotationen: Man spricht in den neuen Bundesländern und in Teilen der doktrinären Linken der Altbundesländer von >Besiegtsein`, diesmal vom >westlichen Kolonialismus«, pflegt Nostalgie (wer erinnert sich da nicht an die berüchtigte salvatorische Floskel >aber Hitler hatte doch auch die Autobahnen gebaut $\measuredangle$ ) und wehrt sich gegen Urbanität und kosmopolitisches, globales Denken, denen man oft genug eine fatale Deutschtümelei, die mit Nestwärme verwechselt wird, ja, ungezügelten Fremdenhaß entgegenstellt.

Aber man muß wohl noch weiter differenzieren. So sind merkbare Unterschiede in der Behandlung der Wende in den Büchern jener Autoren festzustellen, die vor 1989 in den Westen gingen und jenen, die in der DDR verblieben und dort den historischen Zusammenbruch erlebten. Monika Maron und Sigmar Schollak stehen für die Gruppe, die sich schon früher von der DDR abnabelten. Animal triste (Monika Maron) $)^{12}$ und Kallosch (Sigmar Schollak) ${ }^{13}$ artikulieren völlig andere Sentiments, als etwa Volker Braun, Daniela Dahn oder Thomas Brussig. ${ }^{14}$ Eine Zwischenstellung (und Sonderstellung), von der später noch ausführlicher zu sprechen sein wird, nimmt Erich Loest mit Nikolaikirche $e^{15}$ ein. 


\section{Ausgewählte Autoren und ihre Bücher}

Natürlich ist es völlig unmöglich, die Fülle jener Bücher auch nur andeutungsweise zu behandeln, die sich unterdessen zum Thema Wende angehäuft haben. Hier kann es eigentlich nur darum gehen, ein paar Schneisen zu schlagen, ein paar Autoren zu Wort kommen zu lassen, die sich mit unterschiedlichsten Intentionen dem Thema genähert haben.

Vielleicht einer der wichtigsten und dennoch weitgehend unbekannten ist Sigmar Schollak. Hier wenigstens die notwendigsten biographische Daten: Geboren 1930 in Berlin; Musikstudium. Seit den fünfziger Jahren entstanden in der damaligen DDR Kurzgeschichten für Zeitschriften, Texte für den Berliner Rundfunk, Hörspiele usw. Bald Schwierigkeiten mit dem Kulturministerium der DDR, dennoch erschienen insgesamt neun Bücher, darunter biographische um John Brown und Robert Schumann. Noch heute im Handel ist Das Mädchen aus Harrys Straße ${ }^{16} 1982$ reiste er, nach neuen Restriktionen, mit seiner Familie nach West-Berlin aus. 1989 erschien der Band Ausflug in Paradiese ${ }^{17}>$ Geschichten aus Ost und West $\prec$, für die Hans Joachim Schädlich ein Vorwort schrieb.

Freilich sind die meisten seiner Texte, wie gesagt, in der DDR erschienen. Da er dort jedoch ganz und gar nicht zu den staatserhaltenden Schriftstellern zählte, war der Grad öffentlicher Aufmerksamkeit, gemessen an der Zahl von Kritiken in Zeitungen und Zeitschriften, eher kümmerlich. Anders war es schon bestellt um die inoffizielle Aufmerksamkeit, was durchaus so doppeldeutig gemeint ist, wie es sich anhört: einmal um das Interesse nämlich, das die Stasi an Person und Werk des Autors nahm, und zum anderen um die Mundpropaganda, mit der seine Bücher unter Vertrauten in der DDR weiterempfohlen wurden. Was ihm wenig nutzte, als er 1982 in den Westen ging - ein Schritt, der ihn nicht wenig kostete, da die Behörden nach vielem Hin und Her zwar ihn und seine Frau ausreisen lassen wollten, nicht jedoch seinen Sohn. Es bedeutete verdoppelte Repression, als die Familie zusammenbleiben wollte. 
Man kann in seinem neuesten Buch, dem oben schon erwähnten Kallosch, nachlesen, was einer in solch einem Augenblick denkt, da unverhohlen Sippenhaft geübt wird, einer, der schon einmal, unter den Nazis, die Willkür der Behörden, anderer und doch gleicher, kennenlernte und die Zeit lediglich überlebte, weil er snur Halbjude war und seine sogenannt arische Mutter couragiert genug, standhaft zur Familie zu stehen. Den weiter entfernten Verwandten half das nichts, sie kamen in den Lagern der Nazis um, er konnte, mit Glück, das Jahr 1945 erleben.

Wie in einem Spiegel kann man die Gedanken, die Empfindungen, auch die Tatsachen selbst in diesem >Roman einer Autobiographie nachlesen. Kallosch ist spiegelbildlich \Schollak«; Spiegel spielen eine große Rolle in dem Buch, der Held sieht sich in ihnen agieren, sein alter ego ist zwar sehr von dieser Welt, aber zugleich eine Figur, die auch ganz gut in das Universum eines Lewis Carroll paßte. Ein Sonderling in den Augen mancher Betrachter, ein skurriler Mahner, ein spinnerter Typ, der den antiquierten Standpunkt vertritt, ein wenig mehr Moral täte der Gesellschaft ganz gut und sie demzufolge denn auch - natürlich in den unpassendsten Augenblicken - einfordert.

Etwa bei der Wiedervereinigung, und damit sind wir beim Thema: Denn möglicherweise wird der eine oder andere gerade bei der Behandlung der Wende durch Sigmar Schollak Vorbehalte haben. Nur wird er sich dann gegen unbequeme Meinungen, besser: moralischethische Wertungen wenden müssen. Er wird vielleicht Anstoß nehmen daran, daß Sigmar Schollak sich Gedanken darüber macht, ob sein Empfinden, die gewesene DDR und das gewesene >Dritte Reich 〈 hätten mehr Gemeinsamkeiten, als unsere Schulweisheit uns nahe bringt, nicht mehr ist, als eine literarische Provokation und die Wahrnehmung eines, der in beiden Staatsgebilden Verfolgung erlitten hat. Er ist in der Hinsicht rigoros: Was ist denn der Maßstab für das Urteil über eine Ideologie, einen Staat, eine gesellschaftliche Ordnung - die von ihr selbst gesetzten Postulate, ihr selbstentworfenes Bild, oder sind es die Menschen, die in ihnen leben und bestimmte Vorstellungen von ihm entwickelten? Natürlich haben Nationalsozialismus und die Marxsche Utopie ideologisch sehr wenig miteinander gemein - das hinderte sie jedoch nicht, etwa zur Durchsetzung ihrer Heilsvorstellungen glei- 
chermaßen Lager zu bauen, in denen Menschen auf zwar unterschiedliche, auch nicht vergleichbare, dennoch grausame Art zu Tode kamen. Keine Aufrechnung, keine Bagatellisierung, ein nachdenkliches Fragen aus der Perspektive des Opfers.

〉Es war Treue zu einer Sache, zum Menschen nicht «, beschreibt Sigmar Schollak die Motive eines guten Freundes seiner Romanfigur Kallosch, der sich nach der Wende als jahrzehntelanger Spitzel der Stasi entpuppte. Es mag sein, daß sich in diesem Satz die Quintessenz der unterschiedlichen Auffassungen über das Miteinander in Gesellschaften ausdrückt. Aufmerken läßt ja, daß jede Bürokratie die Sache stets höher stellt als den einzelnen Menschen, was, nebenbei, an Volkes Stimme noch aus DDR-Zeiten erinnert: `Bei uns steht zwar der Mensch im Mittelpunkt, aber nicht der einzelne`. Sigmar Schollak ist der Vertreter einer unterdessen offenbar rar gewordenen Spezies, die so etwas als eher zynisch empfindet. Unbehagen auch nach der Wende ist die Grundbefindlichkeit bei Sigmar Schollak; er sieht die Demokratie in Gefahr angesichts eines Bevölkerungsteils, der keine demokratische Kultur gelernt hat und auf eine in der Bundesrepublik stößt, die ihre Bewährungsprobe noch nicht hinter sich hat, gottlob, so setzt er hinzu. Denn er ist einigermaßen pessimistisch auch hier.

Sigmar Schollak ist dabei durchaus verwandt Monika Maron. Ein paar Daten im Telegrammstil auch hier: geb. 1941 in Berlin, Mutter polnische Halbjüdin; der Stiefvater Karl Maron war späterer DDRInnenminister; Abitur; Fräserin in einem Industriebetrieb; Regieassistentin beim Deutschen Fernsehfunk; SED-Mitgliedschaft; Studium der Theaterwissenschaft und Kunstgeschichte; wissenschaftliche Aspirantin an der Berliner Schauspielschule; Reporterin bei der Zeitschrift Wochenpost; ab 1976 freischaffend; 1981 aufsehenerregendes Debüt mit dem Roman Flugasche ${ }^{18}$ über $>$ die schmutzigste Stadt Europas`, Bitterfeld - die Folge war, daß keines ihrer weiteren Bücher in der DDR eine Druckgenehmigung erhielt. Nachdem sie zwischen 1976 und 1977 ein kurzes Techtelmechtel mit der Stasi, der HV A, der sog. Hauptverwaltung Aufklärung des Markus Wolf hatte, der sie unter dem Namen $>$ Mitsu< einige Berichte lieferte, wurde Monika Maron vom Ministerium für Staatssicherheit überwacht und im 〉Operativen Vorgang〈>Wild- 
sau< erfaßt; 1988 übersiedelte sie von Ost-Berlin nach Hamburg. 1991 erschien ihr vielbeachteter Roman Stille Zeile sechs, ${ }^{19} 1993$ Artikel und Essays unter dem Titel Nach Maßgabe meiner Begreifungskraft. ${ }^{20}$ Seit 1994 lebt sie wieder in Berlin.

Animal triste, ihr neuestes Buch, ist in gewisser Weise ein Roman der Wende, wenn auch nur nebenher. Natürlich ist man anfangs gespannt, ob sich irgendeine Anspielung auf die juvenile Stasiverirrung der Autorin, die Affäre >Mitsu<, findet. Doch diese vordergründige Neugier wird rasch verdrängt durch die Kraft einer Erzählung, die eine ganz und gar archaische, fast verzweiflungsvoll unbedingte Liebesbeziehung schildert. Gewiß: nach der vollständigen Lektüre mag, wer will, in der Rahmengeschichte auch eine Studie über die Mechanismen der Verdrängung und des Vergessens sehen - doch das dürfte eher Konsequenz eines literarischen Kunstgriffs als außerliterarische Berechnung sein.

Diese Rahmenerzählung ist eher simpel: Eine Frau, möglicherweise hundert, vielleicht aber auch erst neunzig >oder sogar noch jünger<, erinnert sich an ihre letzte Liebesbeziehung. Die Erinnerung ist grandios und schmerzlich zugleich, denn >eines Tages, es war im Herbst, das weiß ich genau, ist er gegangen und kam nicht mehr zurück «. Nur ganz allmählich im Prozeß dieser Erinnerungsarbeit wird der Ich-Erzählerin und damit dem Leser klar, daß sich hinter diesem Weggang mehr verbirgt als die scheiternde Beziehung mit einem verheirateten Mann. Nichts spürt man also von jenem wehmütig-verklärenden Blick auf die Erinnerung als >Paradies $`$ (Jean Paul). Aus dem Paradies, als das sich anfangs diese Beziehung darstellt, wird im Blick der Frau mehr und mehr eine Vorhölle, bevölkert von Schimären aus den Bildern eines Hieronymus Bosch.

Eine chimära im übertragenen Sinn, ein Brachiosaurus-Skelett, ist Leitmotiv des gesamten Buches. 〉Ein schönes Tier〈, so finden die IchErzählerin, die dieses exklusive Gerippe im Berliner Naturkundemuseum betreut, und ihr Geliebter gleichermaßen; aber der Leser kann sich - zunehmend im Laufe der gigantischen Rückblende - nicht des Eindrucks erwehren, es sei zugleich auch ein bißchen das traurige Tier des 
Titels und das höllische eines Bosch, das schließlich in der Erinnerung der Ich-Erzählerin abweisend und böse die Zähne bleckt.

Und zwischen all diesen Polen wird eine Liebesgeschichte der neunziger Jahre ausgebreitet, die eher ins Altertum paßt, ins Griechenland der Heroen und der Mythen, als in die Jetztzeit. Sie korrespondiert mit dem Chaos der Nachwendezeit, der ebenfalls schmerzlich bewußt werdenden Fragwürdigkeit von Werten, an die zwei Generationen vierzig Jahre lang irgendwie denn doch geglaubt haben. Am Ende steht der Untergang. Persönliche und gesellschaftliche Schuld versinken in einer Amnesie, die jedoch nicht als gnädig empfunden wird. $\mathrm{Ob}$ da unbewußt das Empfinden von Sünde eine Rolle spielt, wie es sich ähnlich in der so augustinisch-puritanischen Sentenz äußert, aus der der Titel stammt: $>$ Post coitum omne animal trister?

Nun ist das Buch zweifellos nicht ein Hauptwerk von Monika Maron, aber wohl ihr ehrlichstes und persönlichstes. Unübersehbar sind in der Grundtextur die Beziehungen zu ihrem Roman Die Überläuferin. ${ }^{21}$ Doch während dort die autobiographischen Bezüge nur vage erkennbar blieben, sind sie hier unübersehbar, geradezu vordergründig. Die Selbstentäußerung geht an die Grenze dessen, was bei uns noch toleriert wird, und sie wird nur erträglich durch die Selbstironie, die der Autorin eigen ist. Monika Marons Figur lebt einen Konflikt, wie man ihn aus japanischen Filmen kennt, in denen die Beziehungen zwischen Liebe, Sexualität und psychischer wie physischer Gewalt weit ungeschminkter geschildert werden. Mag sein, daß das Gefühl einer sachten Ambiguität auch daher rührt.

Interessant ist in unserem Zusammenhang, wie die Wende in dieses Buch eingebettet ist. Einmal als Voraussetzung der Liebesgeschichte überhaupt: Nur sie beseitigte die geographische Trennung, denn der Partner kommt aus dem früheren Westdeutschland. Sie wird weiter expressis verbis als $\gg$ Befreiung $\prec$ von einer $>$ Verbrecherbande $<$ bezeichnet; doch zum dritten wird sie auch als >labyrinthisch nur, weil sie das persönliche Leben total umstülpt, sondern auch, weil sie Sehnsüchte (etwa nach einem paläontologischen Traumziel in den USA) als letztlich nichtig entlarvt. Als äußerliches Sinnbild tritt die Veränderung der Stadt zu einem Straßenlabyrinth in Erscheinung. 
Wie schon eingangs erwähnt, gibt es vergleichsweise wenige Bücher von genuin aus der alten Bundesrepublik stammenden Autoren, die sich mit dem Thema Wende beschäftigen bzw. in denen sie überhaupt eine Rolle spielt. Grass mit Ein weites Feld ${ }^{22}$ natürlich, doch das ist ein so besonderer Fall, der eine gesonderte Untersuchung verdiente, daß hier nur der Titel genannt werden soll. Friedrich Christian Delius mit seinen Die Birnen von Ribbeck, ${ }^{23}$ Hans Christoph Buch mit seiner $>$ deutschen Geschichte< Der Burgwart der Wartburg, ${ }^{24}$ in der er - noch vor Günter Grass und in gänzlich anderer Manier - Hans Joachim Schädlichs Romanfigur >Tallhover aus dessen gleichnamigem Roman adaptierte. Ein jüngeres Beispiel findet sich in Christa Schmidts zweitem, anfangs erwähnten Roman Rauhnächte. Christa Schmidt wurde 1959 in Duisburg geboren, sie lebt seit 1980 in Berlin. Dort arbeitete sie mehrere Jahre als Redakteurin, Lektorin und Rundfunkautorin. Ihr erster Roman, Die Wahlverwandten, ${ }^{25}$ brachte ihr zwar recht gute Kritiken ein, er blieb jedoch in den Buchhandlungen unbeachtet. In Rauhnächte ist die Wende vor allem der Fall der Mauer und damit die Beseitigung von Trennendem. Der Titel avisiert Märchenhaftes, denn >Rauhnächteく, auch $>$ Rauchnächte`, so geben die Lexika Auskunft, liegen in der für den Volksglauben besonders bedeutsamen Zeit zwischen Thomastag (21. Dezember) und Dreikönigstag (6. Januar). Andere zählen dazu nur die `Zwölf Nächte zwischen Weihnachten und dem Dreikönigsfest.

Sonderbarerweise erzeugte das Buch bei mir ein Gefühl der Fremdheit. Obwohl ich die Gegenden der Geschehnisse an der früheren Nahtstelle zwischen Ost und West kenne, oft die gleichen Wege gegangen bin wie die Protagonisten der verwickelten Geschichte, meinte ich immer wieder, in einer anderen Stadt zu leben, andere Leute zu kennen, andere Probleme zu haben. Nur wenigen schließlich dürfte die Frage essentiell geworden sein, ob eine verschwundene, völlig unbekannte Frau vielleicht so etwas wie die Wiedergängerin einer Figur des Malers Caravaggio sein könnte.

Die Konstellationen sind einigermaßen apart, der Gefühlshintergrund, vor dem alles spielt, sehr westlich, vielfach geprägt von den freischweifenden Ideen der Alt-68er. Die Hauptperson, der IchErzähler, ein Arzt namens Selov, wird von seiner Frau Knall auf Fall 
zur Zeit des Endes der DDR aus dem gemeinsamen Haus auf die Straße gesetzt, worauf er sich in Kreuzberg eine Fabriketage mietet, die gerade frei ist und sofort bezogen werden kann. Dort nämlich ist eine Frau mit dem außergewöhnliche Namen Fillede ausgezogen, unter Hinterlassung ihrer ganzen Habe, die sie den Vermieter gebeten hat, mit der Miete der nächsten Zeit zu verrechnen. Während Selov noch etwas ratlos vor dem künstlerisch-kunstvollen Kram der Vorgängerin steht, taucht eine ehemalige Freundin der Verschwundenen auf, Lena, die im Laufe der 254 Seiten des Romans bröckchenweise vom Leben und Treiben der ehemaligen West-Berliner Journalistin berichtet. Besonders von deren Beziehung zu Wörlitz, der in der DDR wohnte, offenbar ein Dauervisum besaß, das jedoch jählings nicht verlängert wurde, so daß Fillede wie eine zeitgenössische Penelope beschloß, auf ihn zu warten. Denn sie durfte andererseits nicht einreisen, weil sie bei der falschen Zeitung beschäftigt war, einer stadtbekannt linken. Natürlich gibt das verschiedenes Kuddelmuddel, weil dann doch jemand anderes auftaucht, $>\mathrm{M} \prec$, ein zunächst anonymer Maler, der Werbeplakate an Berliner Litfaßsäulen und Plakatwänden verfremdet, daraufhin von der Polizei gesucht wird - ein animalisch genialischer Mensch, wesensverwandt besagtem Caravaggio, der ja eigentlich Michelangelo Merisi hieß: eben >M८. Entstehende äußerliche Ähnlichkeiten zu Fritz Langs berühmtem Film $M$ - eine Stadt sucht einen Mörder, sind wohl nicht ganz zufällig. Am Schluß finden sich, so suggeriert es die Autorin, Lena und Wörlitz, und Selov telefoniert selig mit Fillede, die in das Land ihrer Kindheit, Italien, gefahren war. Das Happy-End ist mit beiden Händen zu greifen. Tableau.

Immerhin lernen die Leser eine ganze Menge, so schon nicht über die Stadt, ihre Menschen und Probleme in der Nachwendezeit, dann doch wenigstens über Caravaggio, eine in der Tat beeindruckende Gestalt. Aber wahrscheinlich wäre in dieser Hinsicht das Studium einer einschlägigen Monographie ergiebiger gewesen. Zunehmend im Laufe der Lektüre beginnt man zu argwöhnen, der Autorin wäre der Anfang der Geschichte klar gewesen und vielleicht auch ihr Ende, nicht jedoch der ganze Mittelteil, der ein Buch ausmacht. Nur mühselig schleppt sich der Text voran, ein Stückchen Rückblende folgt auf subjektive Erzäh- 
lung, darauf wieder eine mystische Anspielung, die die Aufmerksamkeit wachhalten soll. Letztlich ein ziemlich vergebliches Unterfangen.

Genau genommen ist die Wende also hier nur Folie, mit der die Autorin eine dünne, kolportagehafte Geschichte zu veredeln suchte. Mir scheint aber genau dies eine Komponente zu sein, die für viele Romane westlicher Autoren konstituierend ist - selbst Grass hat ja, genau besehen, ein nur sehr äußerliches Verständnis für all jene komplizierten Vorgänge, die bei der Wende abliefen. Wenn er sich auf den Beifall vieler aus West und noch mehr Ost beruft, dann zeigt das nur, in welchem Maße er Vorurteile bedient und in welch erschreckendem Umfang die allgemeine Unkenntnis verbreitet ist - eine Tatsache, die man freilich weniger den Uninformierten als denen vorhalten muß, denen Informationen zu verbreiten Pflicht sein sollte.

Obwohl auch bei Erich Loest Elemente der Kolportage unverkennbar sind, hat sein Wende-Roman Nikolaikirche denn doch ein ganz anderes Format. 1926 in Mittweida (Sa.) geboren, wurde Loest nach dem Abitur 1944 Soldat, danach Gelegenheitsarbeiter. 1947-50 Volontär u. Redakteur an der Leipziger Volkszeitung, SED-Mitgliedschaft; seit 1950 freischaffender Schriftsteller; 1955/56 Studium am Literaturinstitut >Johannes R. Becher in Leipzig. 1956/57 stand er mit Gerhard Zwerenz u.a. in Opposition zur SED, insbesondere zu ihrer Kulturpolitik; Mitarbeiter auch am Programm des Leipziger Kabaretts $>$ Die Pfeffermühle<. November 1957 Ausschluß aus der SED; dann wegen seiner Ansichten zu demokratischen Erneuerungen verhaftet und zu siebeneinhalb Jahren Zuchthaus verurteilt; 1964 Entlassung auf Bewährung; Veröffentlichungen von Abenteuer- u. Kriminalromanen unter Pseudonym; erst nach der Wende, 1990, wurde das Urteil von 1957 aufgehoben. Von der Stasi im >Operativen Vorgang〈 >Autor〈 >bearbeitet<; 1979 unter Protest Austritt aus dem Schriftstellerverband der DDR. 1981 kam Erich Loest mit einem Dreijahresvisum nach Westdeutschland; er kehrte nach seinem Ablauf nicht in die DDR zurück. Jetzt lebt er in Leipzig und Bonn.

Viele dieser Angaben kann man nachlesen in einem der wichtigsten Bände zur DDR-Literaturgeschichte und DDR-Literatur- und Kulturpolitik überhaupt, seiner Autobiographie Durch die Erde ein Ri $\beta .{ }^{26}$ Andere 
in den Aufarbeitungen seiner Stasi-Überwachung, Der Zorn des Schafes $^{27}$ und Die Stasi war mein Eckermann oder: Mein Leben mit der Wan$z e .{ }^{28}$ Nikolaikirche ist Höhepunkt und vorläufiger Abschluß eines Zyklus zum Thema Leipzig: Völkerschlachtdenkmal ${ }^{29}$ und Zwiebelmuster. ${ }^{30}$

Auch dieser Roman, der in der letzten Phase der DDR spielt, lebt von den intimen Kenntnissen Erich Loests über seine Stadt. Wahrscheinlich kein anderer könnte es sich erlauben, eine Figurenkonstellation zum Ausgangspunkt zu nehmen, die an Groschenromane erinnert, aber bei ihm völlig selbstverständlich wirkt: Der Alte ist General der Volkspolizei, die Tochter im Baudezernat Architektin, der Sohn Hauptmann bei der Stasi. Der Familie sind adäquate Widerparts beigegeben: Dem General Albert Bacher - zu Beginn der Haupthandlung, 1985, schon ein Jahr tot - seine ergebene Frau Marianne, die mal ein Verhältnis mit einem Republikflüchtling hatte, Linus Bornowski. Der Tochter Astrid Protter, die beginnt, sogar körperlich am Zerbröseln Leipzigs zu leiden, steht ein friedlich mitlaufender Ehemann zur Seite, beschäftigt irgendwo in einem der Feld-Wald-Wiesen-Betriebe der DDR. Beide haben eine Tochter Silke, die so ist, wie man sich eine Tochter in der Pubertät vorstellt: frech, intelligent, in genau jenem Maße aufsässig, daß es auch die Erwachsenen noch verstehen können. Und dem Stasi-Hauptmann Alexander Bacher läuft ein himmlisch-schnuckeliges Wesen namens Claudia Engelmann über den Weg, das sich in der Umweltbewegung der DDR engagiert hat. Alle die haben wieder Freunde und Bekannte, darunter jede Menge Pfarrer oder zumindest DDR-Bürger, die sich in der Friedensbewegung innerhalb und außerhalb der Kirche engagieren, ein paar >Inoffizielle Mitarbeiter der Stasi sind auch dabei - prominentester ein Rechtsanwalt Schnuck -, kurz jeder kennt irgendwie jeden im Ländle, alle zusammen bilden sie ein Biotop, das geradezu prototypisch für die gewesene DDR ist.

Man braucht eine ganze Weile, ehe man dahinter kommt, wie Erich Loest es vermeidet, seine Chronik der letzten Jahre in die Niederungen des Kitsches zu lenken. Die Hauptarbeit dabei leistet die Geschichte selbst. Es war keine, in der Pathos und revolutionärer Schwung Regie führten. Wie Loest etwa am Beispiel des Ehemannes Protter exemplifiziert, wurden es bei den Montagsdemonstrationen in Leipzig schlicht 
immer mehr, die sich entschlossen, der Obrigkeit ins Gesicht zu sagen, daß sie sie satt haben, die also einfach mitmarschierten - stets im Kreis herum. Ein Sinnbild, das ebenfalls keiner gewagt hätte sich auszudenken; es war krude Wirklichkeit. Dazu kommen die literarischen Kunstgriffe: Alexander Bacher trennt sich von seinem Engelswesen, weil sein tschekistisches Gewissen und seine Vorgesetzten es ihm so befehlen. Ein Happy-End findet nicht statt. Der Stasi-Hauptmann ist auch kein Schwarzer Mann, Kinderschreck oder dämonischer Bösewicht, sondern einer jener leicht dicklichen Bürohengste, Handlanger der Macht, wie sie, nicht nur in Deutschland, Tradition haben. Er beklagt verdrießlich die zunehmende Schlampigkeit der DDR-Gesellschaft, die allmählich auch auf die Arbeit der Stasi abfärbt. Gemäßigt mokant äuBert er sich über die Beschränktheit mancher Zuarbeiter von der Volkspolizei, und angesichts der Dämlichkeit einiger $>$ Inoffizieller Mitarbeiter könnte er aus der Haut fahren. Seine Vorgesetzten sind zwar sakrosankt, aber über ihre >Laschheit ist er doch tief entsetzt. >Durchgreifen` müßte man seiner Meinung nach, wie damals beim 17. Juni 1953. Doch freilich ist da der große Bruder im Osten, der nicht mehr recht mitziehen will ...

Andererseits sind auch die Gegenspieler von der Opposition keine reinen Lichtgestalten. Die Pfarrer nicken salbungsvoll lieber einmal zuviel als zu wenig und scheinen innerlich müde, manche Kirchenleute kungeln mit Partei und Staat und wollen eher die Institution retten als die Menschen, die die Kirche aber schließlich erst ausmachen. Das sind mehrheitlich die oberen Ränge. Unten werkeln sie wacker am Weinberg des HERRN - nun gut, wenn gelegentlich bei dem einen oder anderen ein Bröckchen Information für die Obrigkeit abfällt, so ist es schon recht, sie ist schließlich von Gott gesetzt. Nur eine Handvoll sind es, die wirklich Respekt abnötigen: Pfarrer, die mutig alle verteidigen, die bei ihnen Schutz suchen, die die Kirchen zu Stätten einer gewaltlosen Opposition machen, die ihre eigenen Interessen ebenso verleugnen wie die mächtigen der Institution Kirche. Loest zeigt, wie schwierig ihre Stellung im Interessenkonflikt war. Der Titel des Buches ist schließlich Programm: Nikolaikirche. 
St. Nikolai ist Synonym für etwas, das über den Staat hinausreicht, und genau daraus bezieht der Roman seine Kraft. Erich Loest protokolliert den Verfall Leipzigs so genau, daß man gelegentlich glaubt, eine veristische Abwandlung von Alfred Kubins Die andere Seite zu lesen. Natürlich ist Loest nicht Kubin, auch fehlt ihm jene schillernde Doppelbödigkeit und glosende Farbigkeit, die diese literarische Vision des großen Grafikers auszeichnen - doch in der Schilderung des Niedergangs, bei der Beschreibung einer Stadt, die ihre Bewohner zunehmend in Hoffnungslosigkeit stößt, da finden sich Momente, die die ganze Ausweglosigkeit einfangen, mit der die DDR zeit ihrer Existenz mal mehr, mal weniger zu kämpfen hatte. Und dennoch ist die Idee Leipzig auf eine eigentümliche Weise unsterblich. Als im Mai 1968 die Leipziger Universitätskirche gesprengt wurde - eine Tat, die seinerzeit die ganze Stadt empörte - wurde, so schreibt Erich Loest, dafür der Turm von St. Nikolai sichtbar: Ein grandioses Symbol für die Vergeblichkeit, Geschichte auslöschen zu wollen.

Doch sonst scheut Loest die großen Gesten. Und das macht diese Schilderung der Tendenzen, die dann zur Wende führten, so eindrucksvoll. Auch der Untergang der DDR war ja keine klassische Tragödie. Sie brach zusammen, so lautlos und jämmerlich, daß kein Epos je daraus entstehen wird. Ihr Fall wirkte wie ein verdientes Fatum, als wolle noch das Ende den von den Nazis eingeübten und in neuem Gewand von der Sowjetunion geborgten Heroismus ad absurdum führen, der am Anfang des >ersten Arbeiter- und Bauernstaates auf deutschem Boden< so barbarisch prunkvoll zelebriert wurde. Und genau diese Stimmung trifft Loest in einigen Passagen seines Buches meisterhaft. Dabei benutzt er Rückblenden, die dokumentieren, wie brüchig, in welchem Maße von institutioneller und persönlicher Gewalt durchlöchert das geistig-ideologische Fundament des Staates DDR war. Eine ausgesprochen hellsichtige Diagnose, wird doch im allgemeinen Gewalt eher als Motiv für inneren Zusammenhalt interpretiert. Freilich könnte man, um im Bilde des Zerfalls zu bleiben, auch von einer staatlichen Osteoporose sprechen, während Staatsrechtler trocken eine schleichende Legitimationskrise feststellen würden. Loests Retrospektiven reichen dabei weit zurück in die Vergangenheit - bis zum Ausgang der Weimarer 
Republik, zur Flucht des damals noch jungen Bacher in die Sowjetunion, bei der er Verrat andeutet. Eine andere Rückblende schildert die Emigration Albert Bachers, der sich den sowjetischen Partisanen angeschlossen hatte, von denen er >zur Bewährung〈, zweifellos aber auch aus stalinistischem Kalkül, dazu mißbraucht wird, deutsche Kriegsgefangene hinterrücks zu liquidieren. Solcherart gehärtet wie der in der DDR sprichwörtliche Stahl Nikolaij Aleksejewitsch Ostrowskijs (1904 - 1936), ${ }^{31}$ läßt derselbe Bacher in den fünfziger Jahren Linus Bornowski, den früheren Freund seiner Frau, in die DDR entführen, wo er dann für Jahre in einem Zuchthaus verschwindet. Persönliche Rache und >revolutionäre Wachsamkeit gegenüber dem Klassenfeind ‘ - es war im sogenannten sozialistischen Lager einfach, beides unter einen Hut zu bringen.

Linus Bornowski ist Fotograf, er wird von Loest für eine Nebenhandlung gebraucht, die das Verhältnis der alten Bundesrepublik zur DDR illustrieren soll. Ohne Häme, aber doch sehr deutlich, reibt er einem gewissen Typus von Intellektuellen, besonders einigen nicht genannten Medien, unter die Nase, daß sie solche Leute wie Bornowski, die bei Fahrten durch und in die DDR vor dem Verfall nicht die Augen schlossen, als letzte >Kalte Krieger〈 betrachteten. >Die Mauer steht, alles ist tief bis ins kommende Jahrtausend zementiert. Du mußt Dich mit den Realitäten in Einklang bringen, mein Junge $<$, doziert wohlwollend sein Chefredakteur. Es war ein Spruch, der so oder ähnlich öfter zu hören war in den achtziger Jahren. Andererseits, wer hätte damals schon irgendeine Wette darauf angenommen, daß am 9. November 1989 die Mauer beginnen würde zu verschwinden - so spurlos unterdessen, daß selbst altgediente Berliner suchen müssen, wo sie 28 Jahre lang stand.

Nun muß man es aber sagen: Genau solche Geschichten überfrachten auch das Buch. Es gibt keinen der grundlegenden, antagonistischen Konflikte der früheren DDR, der nicht irgendwo und irgendwie auftaucht. Oder zumindest beredet wird. Dabei legt Loest Handlungsfäden, die später nicht wieder aufgenommen werden. Es gibt eine interessante Passage, in der die Unterhaltung eines höheren Berliner Stasi-Offiziers mit Rechtsanwalt Schnuck geschildert wird. In gewundener Rede wird dort eine langfristige Strategie bestimmter Stasi-Kreise angedeutet, die 
Oppositionsbewegungen der DDR so zu unterwandern, daß man sie auch später - was heißt später? - steuern könnte. Wer sich daran erinnert, daß dies im Falle Ibrahim Böhme fast geklappt hätte, dankt noch heute dafür, daß die Stasi-Akten zugänglich sind und offen gehalten werden. Loest allerdings läßt den Faden fallen, er taucht am Ende noch einmal kurz auf, als Spekulation, aber wichtig wird er nicht. Schien das Ganze dem Autor doch zu unheimlich? Unheimlich ist es zwar, aber gerade die Erfahrungen Erich Loests auf diesem Gebiet lassen einen wünschen, er wäre etwas ausführlicher geworden.

$\mathrm{Zu}$ warnen ist davor, das Buch als Schlüssel- oder gar Tatsachenroman zu lesen. Loest hat - wie auch etwa in seinen Büchern Völkerschlachtdenkmal oder Zwiebelmuster - ohne Zweifel viel dokumentarisches Material verwendet, aber Handlung und Personen sind weitgehend Fiktion. In Pfarrer Ohlbaum glaubt man Züge von Christian Führer zu erkennen, einem der bekanntesten Pfarrer von St. Nikolai; natürlich taucht auch Kurt Masur auf, aber das sind im Grunde schon fast willkürliche Zuordnungen. Denn bei Loest sind die Figuren mehr Typisierungen, Personifizierungen bestimmter Handlungs- und Denkweisen. Mag sein, daß der Schriftsteller hier noch mit Rudimenten des weiland sozialistischen Realismus zu kämpfen hat.

Was das Buch auszeichnet, sind die kleinen Gebärden, die Miniaturen innerhalb eines Geschehens, das Loest durchaus nicht immer im Griff behält. Als die Repressionen im Gefolge der Montagsdemonstrationen des Jahres 1989 zunehmen, als schon fast willkürlich verhaftet wurde, steckt hinter dem Fenster der Kirche ein Bogen, >darauf ein Mädchenkopf mit struppig steilem Haar, darunter: »Kopf hoch, Mike! Dein Arbeitskollektiv und Deine Sabine«. Oder jene unglaubliche Szene, als Müllmänner die Kerzen vor der Nikolaikirche entfernen sollen, das auch tun - sie aber fein säuberlich in die Simse stellen und neu entzünden.

Das Buch endet mit der Montagsdemonstration des 9. Oktober 1989. Dieser Tag konstituiert für Loest die Wende. Er ist damit einer der wenigen Schriftsteller, die dafür ein präzises Datum setzen. Ein durchaus plausibles nebenbei: Wie wir heute zu wissen glauben, standen seinerzeit der Erfolg der friedlichen Revolution und die weitere Existenz der 
DDR, zumindest für eine Gnadenfrist, als Alternativen auf dem Plan der Geschichte. Hätte es damals Schüsse gegeben in Leipzig, wäre es zu einem Blutbad gekommen wie auf dem >Platz des Himmlischen Friedens in Peking, die Historie hätte mit Sicherheit einen anderen Lauf genommen. In seiner letzten Szene schließt Loest auch formal den Roman. Er begann in einem ovalen Raum der Stasi am Leipziger Ring, in dem 1985 beschlossen wurde, die Friedensbewegten, die angeblichen Störer der staatlichen Ordnung, vor allem in den Kirchen, endgültig zu erfassen, um sie im Ernstfall internieren zu können. Das Buch endet in demselben Raum, den nun die Stasi-Offiziere verlassen haben, nachdem feststeht, daß es keine Gelegenheit mehr geben wird, die Demonstranten noch aufzuhalten. Zurück bleibt, betrogener Betrüger, Rechtsanwalt Schnuck. Als der Stasi-General durch die Tür geht, >hätte er gern die Faust zum alten Rot-Front-Gruß gehoben. Aber es wäre lächerlich gewesen einem neuen Mann wie Schnuck gegenüber.r Welch ein Bild: Der Hoffnungsträger abgehalfterter staatlicher Macht: ein einsamer, nutzloser Spitzel. - Nicht unterschätzen sollte man, daß Erich Loest durch eine populäre Fernsehverfilmung dieses Buches einen erheblichen Einfluß auf die öffentliche Meinung in Deutschland ausgeübt hat. Ich kann mich täuschen, aber mir kommt es tatsächlich so vor, als habe gerade das Buch Nikolaikirche zur Entkrampfung der Debatte um die Wende, ihre Chancen und Gefahren einiges beigetragen.

Andere Autoren äußern sich versteckter zum Thema: Elke Erb etwa mit ihren >Auskünften in Prosa<, die den sonderbaren Titel tragen Der wilde Forst, der tiefe Wald. ${ }^{32}$ Sie gehört zweifellos zu den wichtigen Autoren in Deutschland. Ihr Lebenslauf ist einigermaßen exemplarisch: 1938 wurde sie in Scherbach (Eifel) geboren, der Vater war wissenschaftlicher Schriftsteller; 1949 Übersiedlung in die DDR; 1957 Abitur; 1957/58 Studium der Pädagogik, Geschichte und Deutsch, 1958/1959 Landarbeiterin; 1959-63 Studium der Pädagogik, Deutsch und Russisch; 1963-66 Volontärin und Lektorin beim Mitteldeutschen Verlag, Halle. Seit 1966 freiberuflich, siedelte sie 1967 nach Berlin um. Erste Texte in der Debütreihe >Auftakt` (1968). Elke Erb löste mit der Rezension der Anthologie In diesem bessern Land (Hrsg. Adolf Endler, Karl Mickel, 1966) die sog. Forum-Debatte über Lyrik aus. An- 
fang der 80er Jahre Kontakt zur unabhängigen Friedensbewegung. Mit dem Vortrag > Von Erich Arendt bis Sascha Anderson - die DDR-Lyrik der letzten fünf Jahre < initiierte sie 1981 erneut eine heftige Diskussion über neue Poesie. Sie war (zusammen mit Sascha Anderson) Herausgeberin der wichtigsten Anthologie nichtoffizieller Literatur in der DDR: Berührung ist nur eine Randerscheinung - Neue Literatur aus der $D D R,{ }^{33}$ die allerdings nur im Westen erscheinen konnte. Über Jahre hinweg arbeitete sie mit an den nichtoffiziellen Zeitschriften Anschlag, Ariadnefabrik, Kontext, Mikado, Radix-Blätter (Wohnsinn), Schaden oder auch Verwendung.

Als direkte Folge der Wende entstand im Oktober 1991 jener Text, der dem Band den Titel gab: Der wilde Forst, der tiefe Wald. Das läßt an Hermann Löns denken, aber Elke Erb zitiert lieber Franz von Schober, Des Jägers Liebeslied von 1826, der auch ganz markige Zeilen beizusteuern vermag: ‘ ... kein Ort, der Schutz gewähren kann, / wo meine Büchse zielt, / und dennoch hab ich harter Mann / die Liebe auch gefühlt<. Man(n) darf gerührt sein. Tatsächlich stehen die knapp sieben Seiten der Titelgeschichte für Inhalt und Machart aller Aufsätze, Erläuterungen, Essays, Reden, Tagebuchnotizen und wirklichen Auskünfte über Lyrik, die zwischen 1989 (einige wenige) und 1995 entstanden. Die Mehrzahl wurde bereits gedruckt, meist allerdings an so entlegener Stelle, daß der Nachdruck in weiten Teilen einer Erstedition gleicht.

Es frappiert, wie hier, in hochdifferenzierter Manier, etwa von der Berichtigung jener Vorurteile erzählt wird, die vielfach mit der Wende aktiviert und jetzt auch in einigen DDR-nostalgischen Kreisen weiter gepflegt werden. Von der Berichtigung des Preußenbildes beispielsweise - und Elke Erb ist viel zu skeptisch, um sich nun andererseits einer Westeuphorie hinzugeben. Das ist übrigens ein Niveau, das Daniela Dahn mit ihrem Essayband Westwärts und nicht vergessen ${ }^{34}$ bei weitem nicht erreicht. Was aber den Band so einzigartig macht, ist die Art ihrer Herangehensweise an das Thema: Elke Erb versucht nämlich, das >Vorher-Nachher`-Schema in vor allem intellektueller, man könnte beinahe sogar sagen: germanistischer Manier zu füllen - ein hochinteressantes Experiment, das meines Wissens ziemlich einzig dasteht. 
$\mathrm{Zu}$ den neusten Büchern, die das Thema Wende thematisieren, gehört Detlef Opitzく Luther-Roman Klio, ein Wirbel um L.. ${ }^{35}$ Ein auch literarisch außerordentlich geistreicher Roman, der auf der Hauptebene Parallelen zum Protestantismus Luthers zieht und auf der Nebenebene, auf der ein Dialog mit einem etwas verdächtigen Herrn Leumull geschildert wird, expressis verbis auf die Wendezeit eingeht. Auch hier allerdings eher in Andeutungen, wenngleich vielleicht etwas offener als auf der Haupterzählebene. Versponnen in seine Forschungen bezüglich Luthers charakterisiert der Ich-Erzähler diese Ereignisse am Ende schlicht als `Quatschく. Kein Leser wird ihm davon auch nur ein Wort glauben angesichts der Überfülle lustvoller Anspielungen auf die Agonie der DDR. Opitz hat Grund für seine Abwehr: Er wurde 1956 in einem Kaff im Erzgebirgischen geboren, Steinheidel, das etwa $15 \mathrm{~km}$ nördlich von Johanngeorgenstadt liegt, und kam schon früh mit der Stasi in unerwünschten Kontakt, die ihn wegen seiner Schreibversuche einsperrte.

Zwei Teile enthält das Buch: Biographische Skizzen zu Luthers Jahrhundert und einen Anmerkungsapparat dazu (Detlef Opitz spricht von >Annotaten $<$ - was auf die DDR zurückverweist, denn nur dort hatte sich noch der Begriff >Annotation<, >erläuternder Vermerk zu einer bibliographischen Anzeige〈, erhalten). Außerordentlichkeit eins: Dieser zweite Teil ist dicker als das eigentliche Buch. Anmerkungen nebst Roman gewissermaßen. Konsequent bis in letzte Einzelheiten versucht der Autor jenen bestimmten Typus der gelahrten romantisch angehauchten Biographie teils zu karikieren, teils aber auch im Ton zu imitieren, der vor allem in den zwanziger Jahren im Schwange war und von der ständigen Überhöhung seines Gegenstandes lebte. (Manchmal freilich schweift die Erinnerung auch zurück an analoge biographische Überhebungen der DDR-Zeit.) Das reicht bis zur Benutzung von Initialen, wie sie ähnlich berühmte Verlage der Vorkriegszeit benutzten und dann schaut man genauer hin und wird inne, daß sie ein neu entworfenes lüsternes Buchstaben-Kamasutra darstellen.

Außerordentlichkeit zwei: Das zweiteilige Buch ist weit mehr als Parodie und Persiflage - Klio, die Muse der Geschichte, wird nicht entblättert, vielmehr zusätzlich mit buntem Flitter behängt. Darunter sind 
die Schraffuren der Luther-Welt sehr sichtbar und deutlich. Doch wo Wahrheit endet und phantastische Ausmalung beginnt, das ist nicht immer auszumachen. Während der etwa zehn Jahre, die Detlef Opitz an dem Buch gearbeitet hat, muß er sich, genau wie Arno Schmidt und mit genau dem gleichen Ziel, eines Zettelkastens bedient haben, der erstaunliche Tatsachen rund um Martin Luther enthält. Opitz erweist sich dabei als ein literarischer Eulenspiegel sondergleichen.

Das Schöne an dieser Prosa ist, daß Opitz mit Luther, dem Kardinal Albrecht, Tetzel und seinen anderen Figuren nichts beweisen will, nichts darstellen, nichts verkünden, nichts kritisieren - außer vielleicht das Pathos einer Zeit, die glaubt, ohne Heroen nicht auskommen zu können -, daß er keine Parallelen ziehen will, sondern einfach nur erzählen. Um so präziser wirken die blitzartig auftauchenden spielerischen Assoziationen zur jüngsten deutschen Geschichte. Es ist erstaunlich, mit welcher Sicherheit Opitz die Sprache der Zeit Luthers beherrscht und abzuwandeln vermag. Ganz kalkuliert setzt er erzählerische Atemlosigkeit gegen die derbe, originäre Sprechweise, die eine gewisse Behäbigkeit auszustrahlen scheint. Das Ergebnis ist eine Lektüre, der man sich nicht einmal beim zweiten Lesen entziehen kann. Es ist unausweichlich, dabei an Umberto Eco zu denken.

Hauptfrage bleibt freilich, wie viele noch Spaß am Spiel mit einer Sprache haben mögen, in die man sich hineinlesen muß, die nicht zum Alltag gehört, sondern Bildungsgut ist, das mehr und mehr untergeht. Es ist Tatsache, daß heute Jüngere nicht einmal mehr Frakturschrift lesen können (und wollen) - wie erst werden sie dann auf diese ungewohnte, starke, gewissermaßen goldgrundige Diktion aus der Lutherzeit reagieren? Unreflektierte Ablehnung wäre doppelt schade, weil sie sich damit einen literarischen $\mathrm{Spa} ß$ mit bemerkenswerten Nebenbedeutungen entgehen ließen, den es nicht allzuoft gibt - gerade in der deutschen Literatur.

Auch Ingo Schramms Fitchers Blau $u^{36}$ hat die Wende als Voraussetzung. Es dauert lange, ehe man erfährt, wie Grimms $>$ Fitcher $〈$ in diesen Erstling des 1962 in Leipzig geborenen (aber im Prenzlauer Berg aufgewachsenen) Schramm gelangt; das Buch spielt vor allem im östlichen Berlin der Nachwendejahre. Kein Blaubart treibt dort verborgen sein 
Unwesen, niemand entführt schöne Schwestern nacheinander in die Einöde - ganz prosaisch wird auf Seite 348 an das Märchen selbst erinnert. Das Erzählen von Fitchers Vogel, das noch mit ein paar unappetitlichen Details angereichert wird, ist Teil der sadistischen Erziehungsmethoden des Unholds Josef am Sohn Karl, der darüber in Verwirrung fällt und fortan als Simplicissimus-Verschnitt durch die Zeiten stolpert. Doch das ist noch nicht alles: Dieser Josef, ein von seiner Gottähnlichkeit zutiefst überzeugter Zeitgenosse, hauptamtlich als Chirurg (vor allem bei der früheren Nationalen Volksarmee der DDR tätig), zeugte, so erfährt man im Laufe des Romans, in der selben Nacht mit zwei verschiedenen Frauen zwei Kinder - besagten Karl und Janni. Sie kommen am selben Tag zu Welt, in derselben Klinik; die Mütter liegen im selben Zimmer und erkennen erst beim Besuch des Vaters die schockierende Kalamität, daß sie demselben Typen aufgesessen sind. Folgen hat das nicht. Doch ich will an dieser Stelle nicht weiter auf diesen literarischen Comic eingehen: Er ist rundum mißlungen - und auf außerordentlich präzise Weise das genaue, sozusagen östliche Gegenstück zu Christa Schmidts Rauhnächte. Die Wende als Folie, vor der eine beliebige Handlung spielt. Was die Ideen der 68er-Generation für Christa Schmidt, sind Wehrdienst und die Erfahrungen schleichender Inhumanität für Ingo Schramm - und das bedeutet natürlich keine inhaltliche Vergleichbarkeit.

$\mathrm{Zu}$ den, nach meinem Verständnis, wichtigen Büchern, die das Thema Wende aufgreifen, gehören Brigitte Burmeisters Romans Unter dem Namen Norma ${ }^{37}$ und ein gerade erschienener Roman von Klaus Schlesinger Die Sache mit Randow. ${ }^{38}$ - Brigitte Burmeister wurde 1940 in Posen geboren. Sie studierte Romanistik in Leipzig und machte dort auch ihren Dr.phil. Von 1967 an arbeitete sie an der Akademie der Wissenschaften in Berlin. Von ihr liegen Veröffentlichungen zum Nouveau Roman vor. Seit 1983 freie Autorin. Veröffentlichungen unter anderen: der Roman Anders oder Vom Aufenthalt in der Fremde ${ }^{39}$ und der ebenfalls zum Thema gehörige Erzählband Herbstfeste. ${ }^{40}$

>Das Vergangene ist nicht tot; es ist nicht einmal vergangen. Wir trennen es von uns ab und stellen uns fremd.< Man darf diesem berühmten Beginn der Kindheitsmuster ${ }^{41}$ von Christa Wolf durchaus die 
ersten zwei Sätze von Brigitte Burmeister an die Seite stellen: >Es ist ein großes Haus, hundert Jahre alt. Der Stadtteil, in dem das Haus steht, hieß weiter Mitte, als er längst Rand war, dahinter Niemandsland, von der Schußwaffe wurde Gebrauch gemacht.<Brigitte Burmeister verdeutlicht damit von Anfang an, daß sie sich nicht fremd stellen will. Diese Sätze rücken aber auch ohne Verzug das große Thema der Wende, die geographische Vereinigung, in der der Stadtteil >Mitte< wieder Mitte von Berlin wird, in den Vordergrund. Zwei große Kapitel nur enthält ihr offenbar auch autobiographisch gefärbtes Buch. Die Titel: Am $>17$. Juni< und >Am 14. Juli<. Es sind große historische Daten, deren Gleichstellung aufhorchen läßt, und zugleich die Tage, an denen die Reflexionen der Ich-Erzählerin Marianne zusammengefaßt werden. Das erste Kapitel, das auf die Insurrektion in der DDR am 17. Juni 1953 verweist, sammelt Impressionen aus dem Berlin nach 1989, setzt sie in Beziehung zur persönlichen Geschichte der Heldin und der mit ihr verbundenen Figuren: von Johannes, ihrem Freund, den es aus >Mitte< nach Westdeutschland zieht, und vor allem von Norma, ihrer Freundin. Es sammelt aber auch Gedanken über die Verhältnisse gänzlich unterschiedlicher Menschen zu deutscher Vergangenheit.

Das zweite Kapitel, das mit seinem Titel an den Sturm auf die Bastille am 14. Juli 1789 erinnert, den jetzigen französischen Nationalfeiertag, berichtet vor allem über den Bruch zwischen Marianne und Johannes. Im Mittelpunkt steht der Anlaß dieser Trennung: die wilde Story, die die Erzählerin der Frau eines Arbeitskollegen von Johannes bei der Einweihungsparty für die geplante neue gemeinsame Wohnung in Mannheim auftischt. Darin schildert sie sich als Opfer der Stasi, gezwungen, bezwungen von einem psychologisch abgefeimten Führungsoffizier; fabuliert sich eine linientreue Familie hinzu, einen ganzen, nach dem Verständnis der >Wessis $<$ : DDR-typischen Lebenslauf - eine aggressive Provokation, ein erbarmungswürdiges, auch erbärmliches Spiel, in dem sie >unter dem Namen Norma als >Inoffizielle Mitarbeiterin agiert. Ein Akt, untauglich zu wirklicher Befreiung, aber gut genug für die Flucht aus einer selbstsicheren, scheinbar in sich ruhenden Gesellschaft, der westdeutschen, der sie hilflos gegenübersteht. 
Für Brigitte Burmeister ist diese Geschichte der subjektiven Andersartigkeit, der Konfrontation mit unbekannten gesellschaftlichen Codes, des immer noch geteilten und teilenden Himmels, Gelegenheit, eine Vielzahl sensibler Beobachtungen und sprachlich äußerst subtiler Bilder auszubreiten. Sie schildert Larmoyanz, ohne dabei selbst in den Ton der Weinerlichkeit zu verfallen. Ganz behutsam schattiert sie Personen und Gegenden von Berlin, besonders um die Marien- und Luisenstraße herum, bis sie vor unseren Augen in einer Frische stehen, als kennten wir sie schon lange. Die Ich-Erzählerin - wie die Autorin Übersetzerin aus dem Französischen - hat bis in kleine stimmige Details Konturen. Dazu gehören die Gespräche mit der erfundenen Tochter Emilia über die Menschen ihrer Umgebung und ihre Entwicklungen. Ein anderes ist die spannungsreiche Beziehung zur Freundin Norma. Ein drittes das sonderbare Eigenleben, das das von $\mathrm{ihr} \mathrm{zu}$ bearbeitende Buch über die französische Revolution zeitweilig zu führen beginnt ist das dort am Tisch nicht Saint-Just? Stoff für den Traum Realität geben auch die Fragmente eines Briefwechsels zwischen Clara Lentz, >seit 1927 Claire Griffith in Amerikaく, wohnhaft im >Haus 2605 am Nido Way in Laguna Beach`, Southern California, und ihren Freundinnen Erna, Ella und Minna in Berlin-Mitte. Ein Briefwechsel, von dem man natürlicherweise nur den amerikanischen Teil kennenlernt, in unbeholfen-klischeehaftem Deutsch: Dokumente, vielleicht sogar echte, einer anderen, so ähnlichen Not, der zunehmenden Vereinsamung in der Fremde und im Alter.

Aber ist nicht Vereinsamung das Hauptthema? In immer neuen Akkorden schlägt Brigitte Burmeister es an, bringt es in Verbindung zur wachsenden Vereinzelung in einer Gesellschaft, die den Sprung in die Effizienz per Crash-Kurs lernen soll - und muß. Daß das dabei auftauchende Leid zum ersten Male glaubwürdig wird, liegt an der scheinbaren Kühle der ästhetischen Mittel. Tatsächlich geschieht hier große Literatur.

Nichts ist da auch von dem Enigmatischen, Labyrinthischen, das Brigitte Burmeisters Erstling, Anders oder Vom Aufenthalt in der Fremde, für manche so schwer verdaulich machte. Und doch ist die Kontinuität unverkennbar: die Erfahrung der Unzugehörigkeit, die Funktionalität 
inmitten des Chaos, die verborgene Determiniertheit, wo von Freiheit geredet wird. Das Verbindende ist die Sprache, die das Personal des Romans nicht nur beschreibt, sondern zugleich auch konstituiert. So, wie sich die Ich-Erzählerin ihre Identität als >IM Norma erfindet, so stecken in den Nebenfiguren auch deren eigene Vexierbilder, Phantasien von Phantasien, Schemen von Schemen von Schemen, die alle gemeinsam erst die Heldin >Marianne Arends, Aufgang B, vier Treppen< ausmachen.

Hier ist, wie ich finde, zum ersten Mal in der Literatur nach der Wende, jener Ton getroffen worden, der diesem Ereignis als einziger gerecht werden kann. Es ist ein Buch der tiefen Menschlichkeit, das die Brüche nicht verkleistert, aber auch nicht unnötig vergrößert. Die Wende ist als Ereignis präsent, das retrospektiv geschildert wird, aber es wird begriffen als Ausgangspunkt für Entwicklungen, denen wir nur begegnen können, wenn wir unsere Erfahrungen nicht verleugnen, sondern uns ihnen stellen.

Genau dies ist auch das Thema des letzten Buches, auf das ich in unserem Zusammenhang noch kurz eingehen möchte: Klaus Schlesingers Roman Die Sache mit Randow. Das Werk ist besonders in einer Hinsicht interessant: Soweit ich es überblicken kann, ist es (von Sigmar Schollaks oben erwähntem Kallosch abgesehen, das jedoch anders determiniert ist) eines der wenigen, die sich nicht scheuen, Parallelen zur Nachkriegszeit zu ziehen und - ganz behutsam - gewisse Verwandtschaften zu konstatieren. Gerade von Klaus Schlesinger hätte man das zunächst kaum erwartet. 1937 wurde er in Berlin geboren, der Vater war Arbeiter; Grundschule; Ausbildung als Chemielaborant. Schlesinger studierte mehrere Semester an der Ingenieurhochschule in WestBerlin und in Fürstenwalde; erste literarische Veröffentlichungen 1960; seit 1964 freier Journalist und Schriftsteller; 1971 erste Buchveröffentlichung (der Roman Michael); 1972 Fernkurs am Literaturinstitut $>$ Johannes R. Becher in Leipzig; seit 1973 Mitglied im Schriftstellerverband der DDR. Er organisierte mit Bettina Wegner 1974 und 1975, bis zum staatlichen Verbot, in Berlin die Veranstaltungsreihen `Eintopp< bzw. >Kramladen<; 1974 Mitinitiator (mit Ulrich Plenzdorf und Martin Stade) der Autorenanthologie Berliner Geschichten, die 1976 nach 
massiven Interventionen des Schriftstellerverbandes und des Ministeriums für Staatssicherheit aufgegeben werden mußte. Seit 1974 wurde Schlesinger von der Stasi observiert (〉Operativer Vorgänge〈 >Schreiberling und `Selbstverlag`); 1976 Mitorganisator einer Solidaritätserklärung zu dem berühmten Protest von 12 Schriftstellern gegen die Ausbürgerung Wolf Biermanns; 1979 war er Mitunterzeichner eines an Erich Honecker gerichteten Briefs von acht Schriftstellern, in dem die repressive Kulturpolitik angeprangert und das Verfahren gegen Stefan Heym wegen Devisenvergehens verurteilt wird; daraufhin im Juni (mit Stefan Heym und sieben weiteren Schriftstellern) Ausschluß aus dem Schriftstellerverband. 1980 Übersiedlung nach West-Berlin; dort engagierte er sich in der Hausbesetzerszene, der Band Matulla und Busch ${ }^{42}$ war Ergebnis dieses Engagements. Seine bekanntesten Veröffentlichungen sind Alte Filme ${ }^{43}$ Berliner Traum ${ }^{44}$ Leben im Winter ${ }^{45}$ sowie Fliegender Wechsel - Eine persönliche Chronik. ${ }^{46}$

Sein neues Buch, Die Sache mit Randow, spielt auf mehreren Zeitebenen. Die aktuelle Handlung bezieht sich auf das Jahr 1991. Darin eingebettet sind Erinnerungen an das Jahr 1951, als die berühmte >Gladow-Bande in Berlin ihr Unwesen trieb, aber damit auch die Kriegszeit, denn ausdrücklich wird in diesen Erinnerungen festgehalten, daß der Krieg und die unmittelbaren Jahre danach den damals Halbwüchsigen präsenter sind als die Gegenwart. Bei Schlesinger heißt Gladow >Randow<; die Halbwüchsigen der Dunckerstraße nannten ihn freilich nur >Ambach<, weil er zur Bekräftigung seiner Sätze an das Ende stets ein >Ambach! ‘ stellte, was immer das bedeuten mochte. Diese Cliquen übten einen erheblichen Gruppenzwang aus, denen sich ihre Mitglieder klaglos unterordneten: der lange Maschke, Benno, Hotta, der Zimmermann, Bernie Sowade, Schmiege oder wie sie alle hießen. Auch der halbwüchsige Thomale, gerufen \Tommie`, wußte es nicht anders, der sich vierzig Jahre später an die dramatischen Ereignisse von 1951 erinnert. Randow wurde - wie sein reales Vorbild Gladow, der >Al Capone von Berlin - damals hingerichtet. Nicht einmal die Westpresse zweifelte daran, daß bei dem spektakulären Proze $\beta$, der Raub und Mord sühnen sollte, alles seine Richtigkeit hatte. Warum also die Nervosität aller möglichen Leute, als sich Thomale, eher durch Zufall, Randows 
wieder entsinnt? Oder bildet er sich das nur ein? Denn Thomale ist selbst nicht gerade in bester Geistesverfassung: Seine Frau Therese ist in den Westen ausgereist, er selbst hat seine Tätigkeit als Fotograf für eine Zeitschrift aufgegeben, weil die Spielräume für kritische Kreativität immer kleiner wurden. Und 1989, als der Schatten Randows wieder emportaucht, sind sowieso alle nervös. Die Ereignisse stehen auf des Messers Schneide, die Stadt brodelt von unterdrückter Erregung, Diskussionen an allen Ecken und Enden ... Doch in jenem Mikrokosmos, in den das Schicksal Randows eingebunden ist, stecken auch die Keime größerer - gegenwärtiger wie zukünftiger - Entwicklungen. Eine sanfte Melancholie liegt über Klaus Schlesingers Liebeserklärung an Berlin. Aber er geht deshalb nicht in literarisch grauen Gewändern - im Gegenteil. Seine Geschichten sind farbig, gutgelaunt, selbstironisch, mit ein bißchen Distanz zu den frühen Jahren der DDR, aber voller Liebe zum Detail eines Lebens auf den Straßen von Berlin. Vornehmlich natürlich auf der Dunckerstraße; und da liegt die Bernauer Straße, jenes seinerzeit weltweit bekannte Synonym für die Mauer, gerade um die Ecke. Klaus Schlesingers Roman ist jedoch mehr, ein Krimi beispielsweise. Man kann sein Buch auch lesen als Studie über die Möglichkeiten, eigene Schuld zu verdrängen, als eine Schilderung von Solidarität, Feigheit, Opportunismus, Mut und Flucht, als eine dramatische Darstellung jener weltstürzenden Tage, da das Gebilde DDR sang- und klanglos zugrunde ging - und nicht zuletzt als eine autobiographisch gefärbte Erinnerung an eine Jugend ohne Fernsehen. Daß sie zu besseren oder schlechteren Menschen heranwuchsen als heute, kann freilich daraus nicht abgeleitet werden. Doch zuallererst ist der Roman wahrscheinlich eine Selbstvergewisserung jener frühen gemeinsamen Geschichte Berlins, die heute über dem Trennenden vernachlässigt wird.

Die Wurzeln für Versagen, den Verfall eines Staates, das Zerbrechen von Hoffnungen, liegen tief in der Vergangenheit. Klaus Schlesinger gräbt sie aus, ohne anklagend den Finger zu heben, ohne Schuldzuweisungen, aber auch ohne jenen Fatalismus, der sich mit der Meinung begnügt, daß es >ja so habe kommen müssen<. Aus den kleinen, persönlichen Verstrickungen erwachsen die großen politischen - so wie umgekehrt private Entscheidungen abhängen von den Gegebenheiten 
des allgemeinen Lebens. In der gleichen Weise, wie in dem Roman aus der Suche nach der Erinnerung an die Flucht Ambachs/Randows/Gladows und sein Ende eine Geschichte erwächst, die mit Mord und Totschlag schließt und noch vierzig Jahre später einen Verrat aufdeckt, genau so erwuchsen aus kleinen, oft als läßlich angesehenen Sünden des Staates DDR an seinem Anfang die großen, historisch unentschuldbaren Inhumanitäten, die unzähligen Menschen die Existenz oder gar das Leben kosteten. >Was bei Windstille schwelt, entzündet sich bei Sturm<, sagt Thembrock, ein etwas anpassungsfähigerer Journalistenkollege von Thomale in diesem Buch, und er setzt noch hinzu, >daß eine Gesellschaft sich immer nur die Fragen stelle, die sie auch beantworten könne . In dem Sinne hat Klaus Schlesinger jetzt die richtigen Fragen gestellt.

Was besticht an diesem Buch und es aus einer ganzen Reihe anderer heraushebt, ist - neben der formalen Geschlossenheit - der weite historische Horizont, den Schlesinger eröffnet. Er weist, soweit das in einem Roman überhaupt möglich ist, nach, daß die Wurzeln für den Zusammenbruch der DDR, für die Wende also, schon in der Vorvergangenheit der DDR liegen. Bei ihm sind die handelnden Personen völlig unbeleckt von der stets beschworenen sozialistischen Aufbruchsstimmung der frühen Jahre, im Gegenteil. Sie beobachten die Anstrengungen des Staates, sich zu konsolidieren, mit Spott und versuchen das beste für sich selbst aus den Zeitläuften zu machen. Schlesinger blättert dabei auch ein weithin unbekanntes Kapitel auf: die organisierte Schmuggeltätigkeit der DDR in den fünfziger Jahren (sie wurde erst 1956 eingestellt). Die DDR tauschte neu gedruckte Ostmark in West-Berliner Wechselstuben in Westmark um (was den Kurs manchmal rapide verfallen ließ), kaufte damit zollfreie Waren, vornehmlich Zigaretten, etwa im Rotterdamer Freihafen ein und schleuste die dann wieder nach West-Berlin, um sie dort mit Profit zu verhökern. Natürlich eine Milchmädchenrechnung sondergleichen, zumal viele Waren in der DDR versickerten - doch Schlesinger zeigt, wie sich daraus eine ambivalente Haltung gegenüber dem Gesetz entwickelt. Wobei das natürlich nur eine Komponente unter vielen ist. 
Die Wende selbst wird bei ihm auf den 9. November 1989 datiert, allerdings scheint mir das eher aus der literarischen Konstellation, der Ortswahl, zu resultieren als aus politischen Überlegungen - zumindest ergibt sich das aus der Nachbarschaft der Dunckerstraße zur Bernauer und andererseits zum Übergang Bornholmer Straße. Denn vielfach ist bei ihm die Rede von den Prozessen, die das Leben ausmachen; überhaupt lebt seine Schilderung dieser Tage von ständiger geistiger Bewegung. 


\section{Pessimistischer Versuch eines Resümees}

Eine Zusammenfassung? Sie könnte vielleicht Theoretiker befriedigen, aber an den Realitäten der Literatur ginge sie mit Sicherheit vorbei. Die Entwicklung ist in einem Maße in Fluß, wie es stets nach großen, welthistorischen Ereignissen der Fall ist. Wobei ich nicht die Vereinigung Deutschlands meine, sondern jenen geschichtsformenden Zusammenbruch der kommunistischen Utopie, die in vielfältigen Wechselbeziehungen steht zu jener Wende in Deutschland, die wir hier thematisieren. Das Buch, das diesen Ereignisketten gerecht würde, ist noch nicht geschrieben, in pessimistischen Stunden bezweifele ich, ob es überhaupt je geschrieben werden wird; ob nicht vielmehr die wirklichen historischen Ereignisse jetzt ganz woanders ansetzen werden, so daß unsere dramatischen Ereignisse später nur als Appendix einer neuen Welthistorie aufscheinen werden.

\footnotetext{
${ }^{1}$ Nicht aufnehmen konnte ich in diese Übersicht Günter de Bruyns Fortsetzung seiner Autobiographie, die unter dem Titel Vierzig Jahre - Ein Lebensbericht (Frankfurt a.M., 1996) erst nach der Tagung in Vlotho erschien.

2 Christa Schmidt, Rauhnächte, Berlin 1996.

${ }^{3}$ Irina Liebmann, In Berlin, Köln 1994.

${ }^{4}$ Volker Braun, Der Wendehals, Frankfurt a.M. 1995.

${ }^{5}$ Marion Titze, Unbekannter Verlust, Berlin 1994.

${ }^{6}$ Wolfgang Hilbig, »Ich«, Frankfurt a.M. 1993.

7 Göttinger Sudelblätter ,hg. von Heinz-Ludwig Arnold, Göttingen 1994.

${ }^{8}$ Uwe Kolbe, >Offener Brief an Sascha Anderson`, Die Zeit, 22.11.1991. dslb., >Meine Biographie, geschrieben von fremder Hand`, Märkische Allgemeine Zeitung, 17.3.95. dslb., >Mauerschatten, ins Wort verlängert` (gekürzte Fassung eines Vortrags in Lissabon über Zensur und Literatur in der DDR), Neue Zürcher Zeitung, 11./12.2.1996.

${ }^{9}$ Freibeuter 67. Berlin, März 1996.

10 Jens Sparschuh, Der Zimmerspringbrunnen, Köln 1995.

11 Angela Krauss, Die Überfliegerin, Frankfurt a.M. 1995.

12 Monika Maron, Animal triste, Frankfurt a.M. 1996.

13 Sigmar Schollak, Kallosch, Berlin 1995.

14 Thomas Brussigs Buch Helden wie wir, Berlin 1995, erscheint, aller Medieneuphorie zum Trotz, vom literarischen Standpunkt her jedoch eher belanglos.

15 Erich Loest, Nikolaikirche, Leipzig 1995.

16 Sigmar Schollak, Das Mädchen aus Harrys Straße, Berlin 1978.

17 Sigmar Schollak, Ausflug in Paradiese, Berlin 1989.

${ }^{18}$ Monika Maron, Flugasche, Frankfurt a.M. 1981.
} 
${ }^{19}$ Monika Maron, Stille Zeile sechs, Frankfurt a.M. 1991.

${ }^{20}$ Monika Maron, Nach Maßgabe meiner Begreifungskraft, Frankfurt a.M. 1993.

${ }^{21}$ Monika Maron, Die Überläuferin, Frankfurt a.M. 1986.

22 Günter Grass, Ein weites Feld, Göttingen 1995.

23 Friedrich Christian Delius, Die Birnen von Ribbeck, Berlin 1991.

${ }^{24}$ Hans Christoph Buch, Der Burgwart der Wartburg, Frankfurt a.M. 1994.

25 Christa Schmidt, Die Wahlverwandten, Berlin 1992.

${ }^{26}$ Erich Loest, Durch die Erde ein Riß, Hamburg 1981.

27 Erich Loest, Der Zorn des Schafes, Künzelsau und Leipzig 1990.

28 Erich Loest, Die Stasi war mein Eckermann oder: Mein Leben mit der Wanze, Göttingen 1991.

29 Erich Loest, Völkerschlachtdenkmal, Hamburg 1984.

${ }^{30}$ Erich Loest, Zwiebelmuster, Hamburg 1985.

31 Nikolaij Aleksejewitsch Ostrowskijs autobiographischer Roman Wie der Stahl gehärtet wurde gehörte zur obligaten Schullektüre in der DDR.

32 Elke Erb, Der wilde Forst, der tiefe Wald, Göttingen 1995.

33 Elke Erb/Sascha Anderson, Berührung ist nur eine Randerscheinung - Neue Literatur aus der DDR, Köln 1985.

34 Daniela Dahn, Westwärts und nicht vergessen, Berlin 1996.

35 Detlef Opitz, Klio, ein Wirbel um L., Göttingen 1996.

36 Ingo Schramm, Fitchers Blau, Berlin 1996.

37 Brigitte Burmeister, Unter dem Namen Norma, Stuttgart 1994.

38 Klaus Schlesinger, Die Sache mit Randow, Berlin 1996.

39 Brigitte Burmeister, Anders oder Vom Aufenthalt in der Fremde, Berlin 1987/Darmstadt 1988.

${ }^{40}$ Brigitte Burmeister, Herbstfeste, Stuttgart 1995.

${ }^{41}$ Christa Wolf, Kindheitsmuster, Berlin und Weimar 1976.

42 Klaus Schlesinger, Matulla und Busch, Rostock 1985.

43 Klaus Schlesinger, Alte Filme, Frankfurt a.M. 1976.

44 Klaus Schlesinger, Berliner Traum, Rostock und Frankfurt a.M. 1977.

${ }^{45}$ Klaus Schlesinger, Leben im Winter, Frankfurt a.M. 1980.

${ }^{46}$ Klaus Schlesinger, Fliegender Wechsel - Eine persönliche Chronik, Frankfurt a.M. 1990. 